Ann. Biol. anim. Bioch. Biophys., 1977, 17 (4), 523-530.

\title{
Etude de l'électromyogramme global chronique de la paroi intestinale du veau préruminant : migration des phases d'activité régulière et relation avec le transit
}

par C. DARDILLAT, Eva MARRERO*

Station de Recherches sur l'Elevage des Ruminants, I. N. R. A. Theix, Saint-Gènes-Champanelle, 63110 Beaumont - France

Summary. Electromyographical analysis of preruminant calf intestine. Relation between migrating activity front and transit time.

The electrical activity of the small intestine of 3 conscious preruminant calves was recorded using 8 to 16 groups of permanently implanted electrodes; it was then related to transit time.

As in fasting dogs (Szurszewski, 1969), fed calves showed a cyclically recurring, caudally migrating band of intense action potential activity, the activity front. This activity front lasted from 2 to $6 \mathrm{~min}$., had a mean period of $40 \mathrm{~min}$., migrated distally from the duodenum to the ferminal ileum in $150 \pm 24 \mathrm{~min}$. (75 measurements). The mean velocity measured at 8 successive points of the intestine was $29,25,20,14,12,9,8.5$ and $7.5 \mathrm{~cm} / \mathrm{min}$.

Sometimes, and especially after feeding, the activity front migrated only along a variable length of the intestine and there was a wide variation (1 to 2) in migration speed at every point.

We measured transit time by the length of time elapsed between the injection of carmin or carbon black by a catheter in the duodenum, and the first appearance of this marker at the ileal fistula. In all 20 experiments, the transit time $(159 \pm 42 \mathrm{~min}$.) and migration time ( $135 \pm 43 \mathrm{~min}$.) were more or less similar, but the relationship between transit and motility was much clearer if the time between injection and the next activity front in the duodenum was added to migration time. The difference between these data and the transit time was generally less than $12 \mathrm{~min}$.

Feeding did not seem to affect migration or transit time.

In a diarrheic calf we observed little front activity and only very irregular migrations along short lengths of the intestine. Transit time was abnormal, being either shorter or longer.

The transit time in small intestine of preruminant calf depended on the migration of the activity front.

\section{Introduction.}

En étudiant les enregistrements électromyographiques obtenus à partir d'électrodes implantées à différents niveaux sur l'intestin grêle du chien éveillé, Szurszewski

* Adresse actuelle : Centre Nacional de Investigaciones Cientificas (CNIC) Habana, Cuba. 
(1969) a mis en évidence, pendant le jeûne, des phases de forte activité électrique de pointe, apparaissant périodiquement sur le duodénum et migrant lentement jusqu'à la valvule iléocæacale. Ce phénomène a été confirmé par Grivel et Ruckebusch (1972) chez de nombreuses espèces animales et par Ruckebusch, Dardillat, Hatey (1972) chez le veau, non seulement pendant le jeûne, mais quel que soit l'état alimentaire. Cette activité « segmentaire » (Grivel et Ruckebusch, 1972) ou de « front 》 (Code et Marlett, 1975) ou « régulière » (Bueno, Fioramonti et Ruckebusch, 1975) s'intègre dans un complexe de 3 ou 4 phases d'activité électrique d'intensité différente qui migrent parallèlement (migrating myo electric complexe ou MMC) (Code et Marlett, 1975) d'une durée totale de $40 \mathrm{mn}$ chez le veau et indéfiniment répétable. La correspondance entre ces phénomènes électriques ef la pression endoluminale a été démontrée $y$ compris chez le veau (Dardillat et Ruckebusch, 1973). La première phase, de repos (absence de salve électrique) correspond à une absence de variations de pression ; la deuxième phase (ou activité irrégulière) est caractérisée par une distribution irrégulière de salves d'intensité variable qui correspond à une succession de «pics» de pression évoquant des ondes propulsives, la troisième phase (ou activité régulière) est la plus caractéristique : elle est composée d'une succession régulière de salves d'intensité maximum correspondant à une élévation de la pression moyenne surchargée de pics de pression. Cette phase est, soit suivie de la phase de repos chez le veau, soit suivie d'une phase d'activité décroissante, la séparant de la phase de repos (Code et Marlett chez le chien).

La significarion de ces phénomènes sur le transit a été abordée par radiographie (Code et Schlegel, 1973) ou par scintillographie (Summers et Helm, 1974) chez le chien. Ces auteurs montrent que les phases d'activité irrégulière et régulière ont un rôle propulsif, l'activité régulière ayant plus particulièrement un effet de balayage. Chez le mouton et le chien, Bueno, Fioramonti, Ruckebusch (1975) constatent par des mesures de transit de marqueur sur un segment intestinal que la propulsion du contenu s'effectue pendant la phase d'activité irrégulière et présument que la phase d'activité régulière assure un barrage amont contre d'éventuels reflux. Par débitmétrie électromagnétique, nous avons montré chez le veau (Dardillat, 1975) que la propulsion du contenu, dans l'intestin grêle, était assurée au cours, et surtout vers la fin, de la phase d'activité irrégulière.

Pour confirmer cette hypothèse, nous avons comparé le temps de transit de marqueurs colorés ef le temps de migration des MMC entre le pylore et la valvule iléocæcale chez les mêmes veaux au stade préruminant.

\section{Matériel et méthodes.}

Sur 3 veaux mâles FFPN âgés de 15 jours, de 35 à $40 \mathrm{~kg}$, nous avons mis en place 16 groupes de 3 électrodes, régulièrement répartis de l'antrum à la valvule iléocæcale, un cathéter sur le duodénum et une canule simple au niveau de l'iléon. La nature des électrodes, la technique opératoire et le matériel d'enregistrement ont été décrits antérieurement (Dardillat et Ruckebusch, 1973).

Ces veaux recevaient, à 9 h 00 et à 17 h 00,3 I de lait reconstitué. Les enregistrements électromyographiques ont été poursuivis sans interruption pendant environ 
un mois ef les mesures de transit ont été effectuées trois ou quatre fois par jour au cours des deux dernières semaines. La position des électrodes a été précisée à l'autopsie.

Pour mesurer le temps de transit duodéno-jéjunal, nous avons utilisé, soił du carmin, soit du charbon animal. Un gramme environ de colorant était injecté dans un cathéter duodénal (dispersé dans $5 \mathrm{ml}$ de sérum physiologique) et nous avons observé sa première apparition dans un sac fixé sur la canule iléale (le contenu de ce sac était ensuite périodiquement réintroduit dans l'intestin). Grâce à l'emplọi successif de ces deux colorants, nous avons pu effectuer des mesures de transit à quelques minutes d'intervalle. Des mesures ont également été effectuées avec du PEG additionné à l'un des deux colorants afin de mettre en évidence d'éventuelles différences de temps de transit.

\section{Résultats.}

Analyse des tracés électromyographiques.

Des ondes lentes, à un rythme déterminé pour un site donné, sont enregistrées en permanence, sauf sur les 20 premiers centimètres du duodénum (fig. 1). Leur amplitude est faible par rapport aux salves de potentiel qui peuvent surcharger les ondes lentes; ceci en raison d'une constante de temps utilisée, inférieure à $0,1 \mathrm{~s}$.

A un niveau quelconque de l'intestin grêle, on observe périodiquement (environ toutes les $40 \mathrm{mn}$ ), une phase d'activité régulière durant 2 à $3 \mathrm{mn}$ sur le duodénum et $6 \mathrm{mn}$, ou plus, lorsqu'on se rapproche de la valvule iléocæacale. Cetfe activité est suivie d'une phase de repos de 2 à $10 \mathrm{mn}$. Elle est précédée d'une phase d'activité irrégulière de 20 à $30 \mathrm{mn}$ (plus brève vers les régions distales). Ces 3 phases qui constituenł un MMC migrent sur toute la longueur de l'intestin grêle dans 70 à 80 p. 100 des cas (fig. 2) et atteignent la valvule iléocæcale en $150 \pm 24 \mathrm{mn}$ (75 mesures). Leur vitesse de migration décroît de l'amont à l'aval avec une remarquable régularité comme le montrent les moyennes calculées à 8 niveaux équidistants sur l'intestin grêle : $29,25,20,14,12,9,8,5$ et $7,5 \mathrm{~cm} / \mathrm{mn}$. Cependant, entre deux sites donnés, la vitesse peut parfois varier du simple au double.

La succession des différentes phases d'activité est moins régulière dans les deux heures qui suivent la prise de nourriture, période où prédomine l'activité irrégulière, en particulier sur la moitié proximale de l'intestin grêle. Des interruptions de migration avec disparition de la phase d'activité régulière, sont observées plus fréquemment pendant cette période.

\section{Mesure de transit et correspondance arec les traces EMG.}

Les temps de transit ont pu être mesurés avec précision parce que le colorant apparaît au niveau de la canule iléale soudainement, à une forte densité. Sa dispersion augmente ensuite progressivement jusqu'à la limite de détection pendant une dizaine de minutes (correspondant à une phase de débit) ef pour un volume émis de $50 \mathrm{ml}$ environ. Dans quelques cas, des traces de colorant persistent encore après une demiheure environ. Si l'on utilise les 3 marqueurs en même temps, les temps de transit mesurés à l'aide de chacun d'eux sont identiques. 

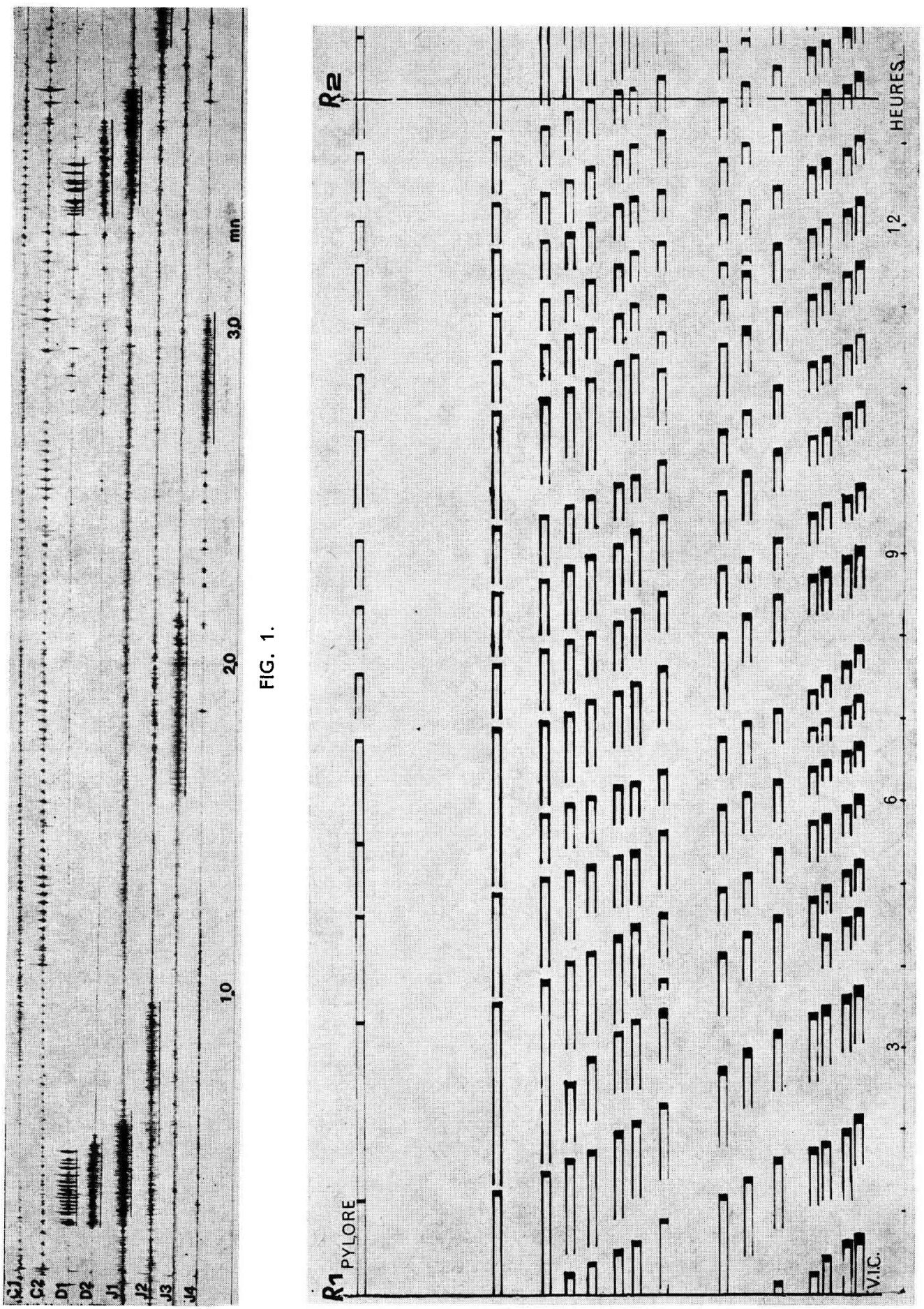

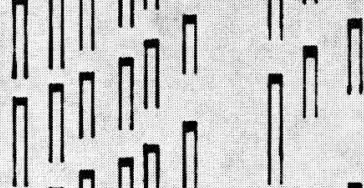
海 (ח 政 - In

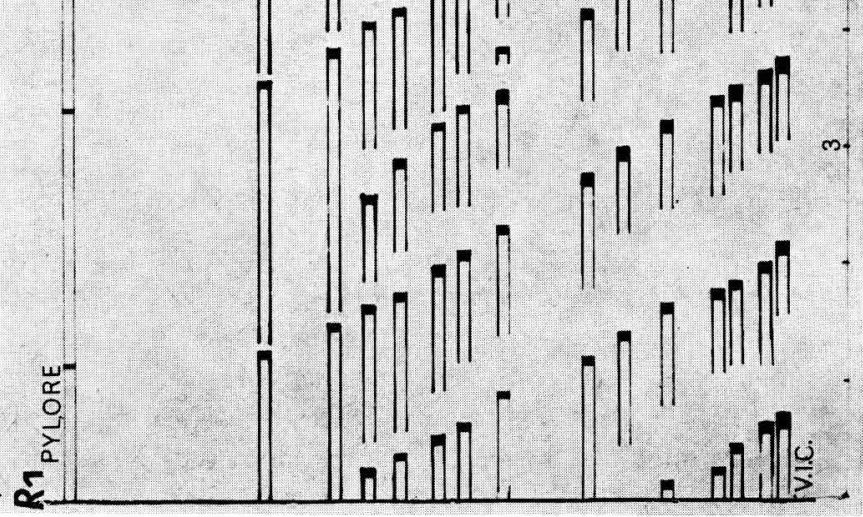


Le transit du marqueur (fig. 3) est sensiblement parallèle à la migration de l'activité régulière qui suit ou précède l'injection du marqueur. L'apparition du marqueur au niveau de la canule précède le plus souvent de quelques minutes l'arri-

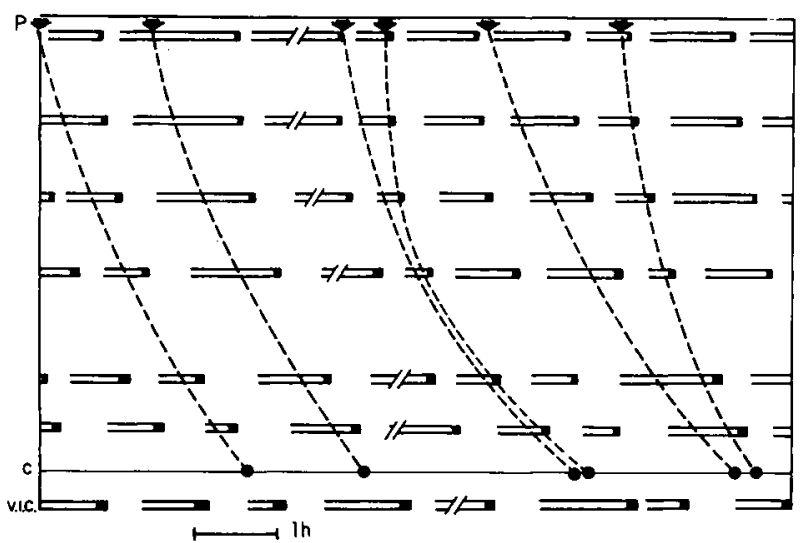

FIG. 3. - Motricité et transit. Ce graphique est construit de la même façon que la figure 2 à partir de 7 groupes d'électrodes duodénales et jéjunales. Des marqueurs différents sont successivement injectés au niveau duodénal $(\downarrow)$ et apparaissent au niveau iléal (•). Le transit est imagé par une ligne pointillée joignant l'origine et l'arrivée du marqueur. La correspondance entre le temps de transit du marqueur et le temps de migration des activités régulières est commentée dans le texte.

FIG. 1. - Enregistrement électromyogrophique effectué sur 1 veau à partir de 8 groupes d'électrodes implantés sur le fondus $C_{1}$, l'antrum $C_{2}$, le duodénum à $5 \mathrm{~cm}\left(D_{1}\right)$ et $15 \mathrm{~cm}\left(D_{2}\right)$ du pylore, le jéjunum à $30\left(\mathrm{~J}_{1}\right), 200\left(\mathrm{~J}_{2}\right), 350\left(\mathrm{~J}_{3}\right)$ ef $500 \mathrm{~cm}\left(\mathrm{~J}_{4}\right)$ du pylore.

Sur le duodénum et le jéjunum proximal, on observe deux phases d'activité régulière, soulignées en noir, d'une durée de 2 à $6 \mathrm{mn}$ et à $35 \mathrm{mn}$ d'intervalle. Elles sont suivies d'une phase de repos (ondes lentes sans salves de potentiel) et précédées d'une phase d'activité irrégulière (salves irrégulièrement réparties). On observe également la migration de la 1 re phase d'activité régulière de $D_{1}$ en $J_{4}$ et de la $2^{e}$ phase de $D_{1}$ en $J_{2}$.

On peut voir sur la caillette une phase de repos ou une diminution de l'activité des salves lorsqu'une activité régulière est présente sur le duodénum mais il n'y a pas à ce niveau des phénomènes périodiques correspondant aux activités régulières.

FIG. 2. - Grophique de motricité construit à portir d'enregistrements électromyographiques effectués simultanément à partir de 15 groupes d'électrodes implantés sur le duodénum et le jéjunum.

La répartition de ces groupes entre le pylore ef la valvule iléocæecale est portée en ordonnée, chaque ligne correspondant à un enregistrement. Les activités régulières, irrégulières et les phases sont représentées par des rectangles noirs, des traits parallèles et des blancs.

Ce graphique montre la succession des phases d'activité ef leur migration sur toute la longueur de l'intestin grêle au cours des $15 \mathrm{~h}$ qui ont séparé le repas du soir $R_{1}$ du repas du matin suivant $\mathbf{R}_{2}$.

On note une fréquence plus faible des activités régulières ainsi qu'une plus grande fréquence d'irrégularités dans la succession ou la propagation des différentes phases sur le 1er tiers de l'intestin grêle au cours des premières heures qui ont suivi le repas. 
vée de l'activité régulière. Les temps de transit les plus longs sont observés lorsque la migration des activités régulières est particulièrement lente, mais surtout lorsque l'injection du marqueur a été effectuée longtemps avant le passage de l'activité régulière. L'activité régulière peut s'interrompre (cf. partie droite de la fig. 3). Dans ce cas, le transit est augmenté de l'intervalle qui sépare l'injection du colorant du passage de la deuxième activité régulière. Le tableau 1 montre que le temps de transit est très peu différent de la somme : temps de migration de l'activité régulière et intervalle de temps entre l'injection et le début de cette activité régulière. Les temps de transit, parfois plus prolongés pendant la phase postprandiale, correspondent à un intervalle particulièrement long dans nos essais.

TABLEAU 1

Correspondance entre le temps de transit d'un marqueur ef le temps de migration des activités régulières

\begin{tabular}{|c|c|c|c|c|c|}
\hline Veau & $\begin{array}{l}\text { Temps écoulé (h) } \\
\text { entre le repas } \\
\text { ef l'injection }\end{array}$ & $\begin{array}{c}\text { Temps } \\
\text { de transit } \\
\text { (mn) } \\
T\end{array}$ & $\begin{array}{c}\text { Temps } \\
\text { de migration } \\
\text { du MMC } \\
(m n) \\
M\end{array}$ & $\begin{array}{c}\text { Intervalle } \\
\text { injection colorant- } \\
\text { départ du MMC } \\
(\mathrm{mn}) \\
1\end{array}$ & $\begin{array}{l}\text { Différence } \\
M+1-T\end{array}$ \\
\hline \multirow[b]{2}{*}{$1\}$} & & $160 \pm \frac{ \pm}{(5)} 11 *$ & $120 \pm 19 *$ & 3 d̀ 61 & $11,2 \pm 9,8$ \\
\hline & $>3$ & $117 \underset{(7)}{ \pm} 19 *$ & $108 \pm 20 *$ & 0 à 45 & $10,5 \pm 7,7$ \\
\hline \multirow{2}{*}{ II } & $<2$ & $189 \pm 20$ & $170 \pm 30$ & 24 à 30 & $8,7 \pm 13$ \\
\hline & $>3$ & $211 \stackrel{(4)}{ \pm} 54$ & $196 \pm 35$ & 6 à 56 & $24 \pm 7$ \\
\hline III & $>3$ & 165 & 143 & 17 & 5 \\
\hline
\end{tabular}

* La canule iléale a été placée à environ $2 \mathrm{~m}$ en amont de la valvule iléocæcale, ce qui peut expliquer des temps de transit et de migration plus courts par rapport à ceux des 2 autres veaux.

Sur un des veaux, pendant une phase de diarrhée spontanée, les MMC ont été très irréguliers et leur propagation impossible à reconnaître. Les temps de transił ont été alors très anormaux, augmentés ou diminués selon les jours.

\section{Discussion.}

La migration des MMC sur l'intestin grêle est actuellement un phénomène bien admis ; cependant, la progression de ces phases sur la totalité de l'intestin grêle d'un préruminant (c'est-à-dire sur près de $20 \mathrm{~m}$ sur nos animaux) est plus difficile à observer que sur l'intestin du chien beaucoup plus court. On pourrait penser que le nombre élevé d'électrodes nécessaires (16 groupes) et la présence d'une canule iléale entraînent une irritation du péritoine qui favorise les irrégularités observées chez le veau par rapport à l'organisation remarquable décrite par Code et Marlett chez le chien (1975). Cependant, sur une vingtaine de veaux étudiés après une inter- 
vention chirurgicale beaucoup moins traumatisante ( 6 à 8 groupes d'électrodes seulement), les mêmes irrégularités électriques ont pu être observées. Ces irrégulariłés sont surtout présentes pendant la phase digestive (fig. 2), alors que les observations de Code sont effectuées obligatoirement sur le chien à jeun.

Dans les études de transit, le choix du marqueur est considéré comme essentiel. On sait en effet que deux marqueurs différents, transitent à des vitesses différentes. De plus, les expériences relatives à la mesure du transit sont réalisées sur l'ensemble du tube digestif qui comprend des réservoirs (estomac, cæcum) dans lesquels le colorant ne se disperse pas toujours de façon homogène. A l'opposé, l'intestin grêle du veau qui ne contient jamais qu'une faible quantité de digesta (moins d'un litre pour $20 \mathrm{~m}$ de long) de nature liquide et homogène explique nos observations selon lesquelles tous les marqueurs transiteraient à la même vitesse.

La correspondance entre le temps de transit ef le temps de migration des activités régulières est un résultat satisfaisant ef s'accorde parfaitement avec les observations de différents auteurs, attribuant un rôle propulsif à l'activité irrégulière, un rôle de balayage à l'activité régulière et une absence de débił pendant la phase de repos. Mais, il est surprenant que chez le chien à jeun, le temps de transit soit beaucoup plus court que le temps de migration des activités régulières (Bueno, Fioramonti et Ruckebusch, 1975). Dans cette espèce, le départ de l'activité régulière coïncide plus ou moins avec l'arrivée à la valvule iléocæcale de l'activité régulière précédente, alors que chez le veau, trois activités régulières sont observées simultanément à trois niveaux de l'intestin (fig. 2). Si la propulsion s'effectue pendant la phase d'activité irrégulière, nos graphiques sur veaux sains montrent que la vitesse de transit est déterminée avec de faibles possibilités de variations, en raison de la fréquence des activités régulières. Il en est tout autrement chez le chien où cette organisation motrice n'impose aucune limite maximum à la vitesse de transit, mais seulement une limite inférieure.

Les observations effectuées pendant une diarrhée où l'on observe simultanément une désorganisation des phénomènes moteurs et des transits anormaux (diminués ou augmentés) autorisent également l'hypothèse selon laquelle l'organisation de la motricité en MMC déterminerait chez le veau le temps de transit dans l'intestin grêle.

Commission CNERNA Digestion-Absorption, Tours, 13 novembre 1976.

\section{Références}

BUENO L., FIORAMONTI J., RUCKEBUSCH Y., 1975. Rate of flow of digesta and electrical activity of the small intestine in dogs and sheep. J. Physiol. London., 249, 69-85.

CODE C. F., MARLETT J. A., 1975. The interdigestive myo-electric complex of the stomach and small bowell of dogs. J. Physiol. London., 246, 289-299.

CODE C. F., SCHLEGEL J. F., 1973. The gastrointestinal interdigestive house keeper : motor correlates of the interdigestive myoelectric complex of the dog. In Fourth Intern. Symp. on Gastrointestinal Motility, p. 63-64. Mitchell Press., Vancouver, Canada.

DARDILLAT C., 1975. In RUTTER J. M. Perinatal ill-health in calves, 111-122. Simpsons Press, Newbury, Berskshire, U. K. 
DARDILLAT C., RUCKEBUSCH Y., 1973. Aspects fonctionnels de la jonction gastroduodénale chez le veau nouveau-né. Ann. Rech. vét., 4, 31-56.

GRIVEL M. L., RUCKEBUSCH Y., 1972. The propagation of segmental contractions along the small intestine. J. Physiol. London., 227, 611-625.

RUCKEBUSCH Y., DARDILLAT C., HATEY F., 1972. La motricité intestinale chez le veau nouveauné. Influence du repas. C. R. Soc. Biol., 166, 1547-1551.

SUMMERS R. W., HELM J., 1974. The effect of feeding and the myoelectric complex on transit in the canine small intestine. Gastroenterology, 66, 132-186.

SZURSZEWSKI J. H., 1969. A migrating electric complex of the canine small intestine. Am. J. Physiol., 217, 1757-1763. 